\title{
Computational Science: An Intermingling of Science, Mathematics, and Computer Science
}

\author{
Frederick R.W. McCourt \\ Departments of Chemistry and Physics, University of Waterloo, 200 University \\ Avernue West, Waterloo, Ontario, Canada N2L 3G1
}

\begin{abstract}
The development of a full undergraduate program in computational science, with both a broad-swath academic plan and four specialization plans, one each in biochemistry, chemistry, earth sciences, and physics, is described. The reasons behind the establishment of the program are presented, and the evolution of the program over the past five years is described. Some of the initial difficulties encountered in setting up such a cross-disciplinary and relatively flexible program of this type are also pointed out.
\end{abstract}

\section{Background Information}

Some background information regarding the University of Waterloo (UW) will be useful prior to discussing our program in Computational Science. UW is what can be referred to as a medium-sized comprehensive university, with six Faculties: Applied Health Sciences, Arts, Engineering, Environmental Studies, Mathematics, and Science. It was founded in 1957 as an engineering school with auxiliary faculty members for the teaching of those arts and science courses required for graduates of the academic programs to meet the specifications required for accreditation as professional engineers. All academic programs were offered only under a concept referred to as 'cooperative education', in which students alternate academic terms of four months duration with 'work terms' of four months duration, during which they are employed by a company or agency participating with the University of Waterloo in one of its 'work-study' programs.

Independent Arts and Science Faculties had evolved by 1960, each offering academic programs, leading to BA and BSc degrees, respectively. They both began by offering programs only in the usual mode of sequential four-month Fall/Winter terms per year; not much later, however, the Science Faculty saw the advantages of offering some of its programs in the cooperative education mode developed by the Faculty of Engineering. It was not for another fifteen or so years that the Faculty of Arts also began offering some of its academic programs in the cooperative mode. Today, the University of Waterloo has over 10,000 coop students, spread over all six Faculties. Graduate programs in most areas of endeavour had also emerged by the mid-1960s.

This new university recognized quite early on that digital computers were going to revolutionize our society and, consequently, in the mid-1960s a decision 
was taken to create a Faculty of Mathematics centered on the discipline of Computer Science, but offering both graduate and undergraduate degrees in all areas of mathematics as well as in the area of computer science. This initiative was a bold one forty years ago: its success can be seen in the approximately 3500 undergraduate students who register annually in the Faculty of Mathematics, with about two-thirds of them working towards a baccalaureate in computer science.

The University of Waterloo is probably best known for its Faculties of Engineering and Mathematics, specifically for their electrical and computer engineering and computer science academic programs, respectively. The Faculty of Science is the fourth largest (or third smallest) Faculty at the University of Waterloo and is made up of four Departments and two Schools: Departments of Biology, Chemistry, Earth Sciences, and Physics, and Schools of Optometry and, most recently, of Pharmacy. Each academic unit has its own degree $\operatorname{program}(\mathrm{s})$; in addition, some units partner with others to offer joint programs, such as Biochemistry, Bioinformatics, Chemical Physics, and Geochemistry, or participate in the broader Faculty of Science programs, such as Honours Science, Honours Science and Business, and Honours Computational Science, via specializations in their core disciplines.

The Computational Science program is the youngest full program in the Faculty of Science and is also one of the youngest programs at the Univeristy of Waterloo. A set of program plans was borne out of the perception that there was a need in modern society for graduates of an academic program in which the combination of a thorough grounding in a conventional area of science, coupled with a significant degree of mathematical sophistication and a strong computer science background, would create graduates who would be prepared to tackle a wide variety of problems arising in today's world.

\section{The UW Computational Science Program}

The Computational Science program at UW has been designed to provide a series of academic plans for students who either wish to attain fairly intensive coverage of one of the traditional areas of science while simultaneously acquiring a broad background in mathematics together with an in-depth knowledge of computer science or who wish a broader but less intensive coverage of all of the traditional areas of science but with the same coverage of mathematics and computer science. The program has four academic plans that focus upon specific traditional areas of science, namely biochemistry, chemistry, earth sciences, and physics, as areas of specialization, plus one academic plan for those who do not wish to specialize in one specific area of science. The Biology Department did not participate in this program as it had already joined forces with the Computer Science Department in mounting a four-year degree program in Bioinformatics.

A typical full program plan at UW consists of forty term lecture courses (each comprised of 33 lectures) plus any other specified laboratory and/or field courses. All Computational Science program plans have a common set of seventeen core 
Table 1. Core courses for all Computational Science program plans

\begin{tabular}{lll}
\hline Faculty & Course Number & Basic Course Content \\
\hline Science & CHEM 120/120L & Properties of matter \\
& CHEM 123/123L & Chemical reaction rates, equilibrium \\
& EARTH 121/121L Natural geological processes \\
& EARTH 122/122L The Earth's history and resources \\
& PHYS 121/121L & Particle dynamics, conservation laws \\
& PHYS 122/122L & Wave motion, gravitation, and heat \\
\hline Engineering & ENVE 224 & Probablilty and statistics \\
\hline Mathematics & MATH 114 & Linear algebra \\
MATH 127 & Differential calculus \\
MATH 128 & Integral calculus \\
MATH 227 & Multivariate and vector calculus \\
MATH 228 & Ordinary differential equations \\
CS 123 & Programming principles using JAVA \\
CS 126 & Principles of software development \\
CS 230 & Computers and computer systems \\
CS 234 & Data types and structures \\
CS 370 & Numerical computation \\
\hline
\end{tabular}

term courses, consisting of six freshman science courses with accompanying laboratories, six mathematics courses, and five computer science courses. This set of courses can be found in Table I. Each student in the program must also select two additional elective junior or senior level computer science courses to complete the basic core component of the program. The remaining twenty-one term courses depend upon the specific plan chosen by a student: however, because of the need to acquire a reasonable depth of knowledge in mathematics and computer science in addition to the basic areas of science, plans in this program contain at most six free electives.

Each specialization plan contains a set of courses that are considered core for the particular specialization, a number of courses from which to select specialization electives, plus a few free electives, while the non-specialized plan has an additional core set of seven chemistry, four earth science, and four physics courses, for a total of fifteen additional core science courses, plus six free electives. The structures of the various plans are shown in Table II.

We believe that the current structures of the Computational Science program plans allow students a reasonable level of flexibility for choosing courses that are of interest to them and to tailor an academic plan that suits their perceived needs. The program is challenging, and has so far attracted a number of very capable, even gifted, students, judging from the number from this program who make the Dean's Honours list each term.

The seven specialization electives associated with the Biochemistry Specialization have been structured to allow students to obtain three-course sequences at the sophomore level and above in at least two of the traditional analytical, 
Table 2. Course structures for Computational Science plans at UW

\begin{tabular}{lcccc}
\hline Plan & \multicolumn{4}{c}{ Number of term courses } \\
& Basic Core & Other Core & \multicolumn{2}{c}{ Electives $^{\text {n }}$} \\
& & & Spec $^{\mathrm{n}}$ & Free \\
\hline No Specialization & 19 & 15 & - & 6 \\
\hline Specialization: & & & & \\
Biochemistry & 19 & 10 & 7 & 4 \\
Chemistry & 19 & 8 & 7 & 5 \\
Earth Sciences & 19 & 11 & 5 & 5 \\
Physics & 19 & 12 & 4 & 5 \\
\hline
\end{tabular}

inorganic, and organic chemistry subdisciplines. Similarly, the seven specialization electives associated with the Chemistry Specialization have been structured so as to ensure that students will complete three-course sequences in physical chemistry plus two of the other three traditional subdisciplines of chemistry.

\section{Growing Pains with the Initial Version of the Program}

Two problems were encountered when this program was first offered in 2001. First, students were being asked to choose a particular plan of study upon entry into their freshman year: this had the consequence that changes in plan were difficult to handle after the first term had been completed, as each specialization plan already contained at least one required specialization core course by the end of the Winter term. Second, the initial version of the program had calculus, chemistry, computer science, and physics/earth science courses in each of the two freshman terms, plus a linear algebra course in the first term and either a free elective or a specialization core course in the second term. This turned out to be the more serious problem for the initial group of 35 students: not only did they find all these courses rather demanding, but also there seemed to them to be almost no overlap of content. Moreover, as they were also adapting to the significant differences between life in high school and university environments, many of the students found the program overwhelming, with the end result being that a number of them fell by the wayside.

To make our Computational Science program more flexible, and at the same time to help students undergo a less demanding adaptation into the university environment, all five plans were rearranged to allow the creation of a common first year. This not only alleviated much of the pressure on the freshman group, but also allowed the decision of whether to specialize or not to be made at the beginning of the sophomore year rather than prior to starting university. At present, all students entering this program share a common first year of study with two term courses each of freshman calculus, chemistry, earth sciences, and physics, and one term course in each of linear algebra and introductory computer science programming principles. Upon completion of this set of common 
introductory courses, students are asked either to choose one of the four areas of specialization or to indicate that they wish to remain in the broader nonspecialized plan.

An ongoing concern for students in this program is the number of timetable conflicts that can arise due both to the cross-disciplinary nature of the program itself and to its crossing of Departmental and, especially, Faculty lines. Because the number of students in the program is still relatively small (roughly fifteen to twenty students in each year of the program) and a smaller still number of students take any particular course following the freshman year, there is not much opportunity to negotiate time changes for lecture courses that are in conflict. This requires the program advisor to be thoroughly conversant with all available antirequisite courses and to be able to suggest and/or approve changes to individual plans to allow similar, though not necessarily equivalent, courses to be substituted as needed in order to facilitate successful completion of a particular student's academic plan.

\section{Some Comments and Observations}

After five years of operation, despite having had to go through a number of growing pains, we have concluded that our Computational Science program can be considered a success. While it does not yet draw as many students as we had originally envisaged, this may be in part due to some of the specific growing pains mentioned above and in part to the fact that we have not been overly aggressive in promoting our program, especially in Ontario high schools. Support for this latter observation comes from anecdotal evidence based upon discussions with students who have transferred into our program from other Faculty programs on the UW campus, during which comments have been made to the effect that had they been aware of the UW Computational Science program when they graduated from high school, they would have entered it directly at that time. This may in large part be because while the Computational Science program has equal billing with Departmental programs (such as those in Chemistry and Physics) in the Faculty of Science brochure sent out to all Science applicants, students who have applied to other Faculties would not normally receive information about this particular program.

We haven't been able to obtain any significant statistics on post-graduate employment or studies as we have yet to graduate a full class from this relatively new program. However, one potentially relevant measure of success of the Computational Science program lies in the success rate for placement of the roughly two-thirds of our students who follow the cooperative mode of study: there have been almost no work placement problems, even though students in our program compete directly for these work-term positions against students from both the Computer Science and Computer Engineering programs at UW. This observation is further corroborated by the level of satisfaction with the performance of our students expressed by the coop work-term employers. Further, we are aware of two of our forthcoming graduates who have applied to 
carry out graduate work in chemistry and physics departments in the USA and in Canada: one has already informed us that he has been accepted by a major Canadian graduate school in chemistry, conditional upon successful completion of his Computational Science with Chemistry Specialization degree at UW. Of course, Computational Science students and graduates have different overall skill sets than do CS students and graduates: on the one hand, they take roughly half the number of CS courses taken by CS students, and on the other hand, they have both a broader and deeper coverage of traditional Science subjects.

It is worth pointing out that a number of computationally intensive courses have been created in the Faculty of Science at the University of Waterloo over the past four years, mainly arising because of the existence of a core of computationally literate students, some of whom are associated with the Computational Science program itself. Courses that fall into this category are: BIOL 366 (Introduction to Bioinformatics), BIOL 382 (Computational Modelling of Cellular Systems), CHEM 355 (Computational Physical Chemistry), CHEM 452 (Computational Quantum Chemistry), CHEM 465 (Molecular Modelling and Protein Folding), PHYS 239 (Scientific Computation 1; numerical analysis applied to physics problems), PHYS 339 (Scientific Computation 2; Monte Carlo and molecular dynamics methods). There are also a number of other courses that have become available through a new complementary Computational Mathematics program introduced very recently by the Faculty of Mathematics. While this newer computationally oriented program has a number of aims in common with our Computational Science program, it has a stronger mathematics focus and does not have the strong science background of our our program. Finally, it should be said that additional computationally intensive courses will be created in the next few years, all of which can enrich the experience of students in our Computational Science program.

\section{Conclusion}

Academic plans of the type offered under the aegis of a Computational Science program should appeal to a select group of modern students who have a desire to couple their interests in science with their fascination for digital computers and the power of numerical procedures and simulation methods. Computational Science provides both a sufficient challenge to good students and a recognition of what can be accomplished when the power of digital computation is combined with in-depth scientific training. 